\title{
On the Importance of Asymmetries in Grasp Quality Metrics for Tendon Driven Hands
}

\author{
Jiaxin L. Fu \\ Robotics Institute \\ Carnegie Mellon University \\ Pittsburgh, PA 15213-3890, USA \\ jfu@cs.cmu.edu
}

\author{
Nancy S. Pollard \\ School of Computer Science \\ Carnegie Mellon University \\ Pittsburgh, PA 15213-3890, USA \\ nsp@cs.cmu.edu
}

\begin{abstract}
Grasp quality measures are important for understanding how to plan for and maintain appropriate and secure grasps for pick and place operations and tool use. Most grasp quality measures assume certain symmetries about the mechanism or the task. For example, contact points may be considered to be independent and identical, or an ellipsoidal measure such as the force manipulability ellipsoid may be used. However, many tasks have strong asymmetries, where wrenches in certain directions dominate. Tendon driven hand designs may also have strong asymmetries, leading to differing abilities to apply contact forces in different directions. This paper begins to explore empirically the validity of some of the symmetry assumptions employed by common grasp quality metrics. We examine the human hand and the Shadow Robot Hand, and find that force abilities vary with finger choice and with location of the contact on the finger for both hands. However, while the human hand shows dramatic changes for different poses due to its asymmetric design, the Shadow hand, with a symmetric design shows much smaller changes and resembles the assumption of identical and independent contact points reasonably well. Thus, we suggest that the underlying design of the hand is a very important factor to consider for grasp quality metrics and for grasp planning and control. The specific grasp quality metric we study in this paper also brings together a variety of previous research. We outline a linear programming approach for computing a grasp quality metric that includes tendon force constraints and contact constraints and can handle any task described as a polytope in wrench space.
\end{abstract}

\section{INTRODUCTION AND BACKGROUND}

Dextrous multifingered robot hands are appealing because of their extreme flexibility. For humanoid robots, they are appealing because of their physical resemblance to human hands. In order to plan grasps for such hands, however, we must have some means of measuring the quality of the results and this quality metric should in many cases account for asymmetries in the task, pose, and hand design. This paper considers a grasp quality measure that takes into account the kinematics and force transmission mechanisms of the hand and allows tasks to be expressed as polytopes of wrenches that must be applied to the object. Empirical results on a human hand model and the Shadow hand show that the hand design and the hand pose should be considered when measuring grasp quality as the ability of the hand to transmit forces to an object.
There has been a great deal of research on developing grasp quality measures based on force and/or stiffness for grasps. Most of this research focuses on selecting good points of contact on the object surface, which is a necessary condition for achieving a good grasp. Many authors make use of an abstract contact model (e.g., [1]-[15]). However, these algorithms typically treat all contact points equally, which may not be an accurate reflection of the mechanical constraints of the hand.

Quality metrics that include hand mechanical constraints, (e.g., joint torque constraints) have also been well studied (e.g., [16], [17], and [18]). However, ellipsoidal metrics, such as force manipulability ellipsoids and ellipsoidal wrench space quality measures are most commonly considered in this research.

To capture a broader range of task asymmetries, Zhu, Ding, and Li [11], Zhu and Wang [12], and Pollard [13] consider tasks as convex polytope in wrench space. To date, this work has focused on contact points that are identical and independent.

Computation of quality metrics where the task is described as a polytope in wrench space often includes as an inner loop a computation to maximize the wrench applied to the object in a given direction (e.g. [11], [12], and [13]). As a result, to extend these quality metrics to accommodate joint torque constraints, we can draw on the vast body of research on wrench optimization. The following research is of particular relevance to this paper: Kerr and Roth [19] reduce the selection of internal grasp forces to a linear programming problem which considers friction and joint torque limit constraints and define the difficulty of the grasp as the minimum distance from the constraints: the larger the minimum distance, the easier the grasp. Melchiorri [18] explores all the nullspaces involved in the analysis of the force systems acting in static conditions for multiple whole-limb manipulation devices. Han, Trinkle, and $\mathrm{Li}$ [20] build on Buss, Hashimoto, and Moore [21] to transform the nonlinear friction cone constraints into positive definite constraints imposed on certain symmetric matrices and formulate force optimization with joint torque constraints as a linear matrix inequality problem.

Tendon driven systems have been considered by a number of researchers for the possibility of achieving humanlike hand 
anatomy and function (e.g., [22]-[26]). For tendon driven hands, it is important to also consider constraints on tendon forces, or muscle activation levels. A number of researchers have explored quality metrics and force capabilities while considering tendon tension constraints. For example, Kurtz and Hayward [27] derive a condition number for a pose of a system with a minimum number of tendons, and Bicchi and Prattichizzo [28] formulate a solution to the problem of identifying tendon tensions to balance an external wrench using stiffness models for the tendons, joints, and contacts.

Force optimization with hand kinematic constraints has also been considered in the area of parallel manipulators (e.g., Nokleby et al. [29]). We can measure quality of a pose using techniques for analysis of parallel manipulators (e.g., measures based on stiffness, mobility, or power transfer functions) as in Woglewede and Ebert-Uphoff [30], although these measures do not handle well directional constraints such as friction cone constraints in a grasp.

Other work that considers quality measures for high degree of freedom mechanisms includes Chinellato et al. [31], who present twelve quality measures for three fingered grasps of polygons and consider fingertip positioning specifically for the Barrett hand; and Zeghloul, Blanchard and Pamanes [32], who determine manipulator placement for any given task by minimizing trajectory travel time, maximizing the distance of the hand configuration from the joint limits and maximizing performance criteria, such as manipulability under specified task conditions.

In this paper, we bring together a variety of this previous research to describe a linear programming approach for computing a grasp quality metric that includes tendon force constraints and contact constraints and can handle any task described as a polytope in wrench space. Using this approach we begin to explore empirically the validity of some of the symmetry assumptions employed by common grasp quality metrics. We examine the human hand and the Shadow Robot Hand, and find that force abilities vary with finger choice and with location of the contact on the finger for both hands. However, while the human hand shows dramatic changes for different poses due to its asymmetric design, the Shadow hand, with a symmetric design, shows much smaller changes and resembles the assumption of identical and independent contact points reasonably well for the grasps that we tested.

\section{Computing the Grasp Quality Measure}

\section{A. Grasp quality metric}

The family of grasp quality metrics considered in this paper is motivated by a desire to capture asymmetries in the task, the grasp, and the mechanism for force based tasks. We also would like a fast solution suitable for use in an optimization loop, for example, and so we formulate grasp quality measurement as a linear programming problem. To make the problem linear, we linearize the contact constraints, and we also assume that the mechanism can actively apply the desired task wrenches. Section IV discusses extensions to lift these restrictions.

Many force-based grasp quality measures are based on minimizing contact forces required to complete a task (e.g. [10], [11], [12], [13], and [15]). A force-based task can be represented generally as a set of wrenches that must be exerted on the object. We represent a task as the set of wrenches $t_{i}, i=1, \ldots, t$ whose convex hull bounds the space of wrenches required for the task as in [12] [13]. We then consider the following grasp quality metric:

$$
Q=\min _{i} \frac{\left\|w_{i, \max }\right\|}{\left\|t_{i}\right\|}, i=1, \ldots, t
$$

where $w_{i, \max }$ is the maximal wrench that can be applied to the object in the direction $i$. By representing the desired space of task forces as a polytope in wrench space, we reduce the grasp quality computation to one of repeatedly finding the maximal wrench that can be applied by the hand to the object in a given direction.

\section{B. Geometry of the applied wrench computation}

This section gives a geometric description of the problem of computing the wrenches that can be applied to a grasped object by a tendon driven system. It is this space of wrenches that we want to match to task requirements to compute a grasp quality metric. Because a geometric approach using the pseudoinverse has been used for similar problems (e.g., [33] and [34]), we begin with a description of the problem geometry and describe the difficulty with applying this geometric approach for a high DOF system.

With a tendon driven hand, wrenches that are actively applied to the grasped object are generated by positive forces exerted along the tendons. Each tendon will have a range of forces it can apply $0 \leq \gamma_{j} \leq \gamma_{j \text {,max }}$. This range of forces is normalized by referring to the tendon's activation level $0 \leq a_{j} \leq 1$. When tendon $j$ has an activation level of 1 , it applies a force $\gamma_{j, \max }$ along the line of action of the tendon.

The set of all possible tendon activations, then, is a unit hypercube $A$ in the space of activation levels, with vertices $a_{i}$ formed from all combinations of maximum and zero activation at the various tendons (e.g., see [33]). Geometrically, this space can be projected into a space of wrenches that can be actively applied to the grasped object by taking the following sequence of steps.

First, map each vertex $a_{i}$ in $A$ to joint torque $\tau_{i}^{\prime}$ :

$$
\tau_{i}^{\prime}=M a_{i}
$$

where matrix $M$ is a linear mapping from tendon activation levels to joint torques, which contains the tendon moment arm and the maximum muscle force information. The space $T^{\prime}$ bounded by the convex hull of the $\tau_{i}^{\prime}$ is the space of joint torques that can be actively applied by the manipulator.

For a static grasp, some combinations of joint torques are not desirable, because they do not produce contact forces, but instead result in self-motion of the fingers. To eliminate these joint torques, $T^{\prime}$ must be intersected with the column space 
of $J^{T}$, where $J$ is the Jacobian mapping forces at the contact points to the torques at the joints:

$$
T=T^{\prime} \bigcap \operatorname{Range}\left(J^{T}\right)
$$

Now contact forces are computed from the space of available joint torques that can produce a static grasp. If torques $\tau_{i}$ bound the space $\mathrm{T}$, then we have:

$$
f_{i}^{\prime}=J^{+T} \tau_{i},
$$

where $J^{+T}$ is the pseudoinverse of the Jacobian transpose. Mathematically there is also a homogeneous term in the right hand side of the equation, which represents the preloading forces, or forces that correspond to zero joint torques. This factor complicates the analysis, as preloading forces are generally not known and cannot be actively controlled [28]. It is not a factor in our experiments, however, because in practical grasps with a fully actuated arm and hand, there will not be contact forces that produce no torque about any joint and thus $J^{T}$ will not have a nullspace. ${ }^{1}$

If the space $F^{\prime}$ is bounded by the convex hull of contact forces $f_{i}^{\prime}$, it must next be intersected with the space of forces allowed with our contact model:

$$
F=F^{\prime} \bigcap \text { Contact ModelConstraints. }
$$

Finally, the space of available forces can be mapped to the space of wrenches that can be actively applied to the object. If we linearize the contact constraints, $f_{i}$ can be found as bounding points of $F$, and the space of external wrenches $W$ is bounded by $w_{i}$ :

$$
w_{i}=G f_{i}
$$

where $\mathrm{G}$ is the grasp matrix.

The geometric computation outlined here can be very time consuming due to the subspace intersections that are required as part of the process. For example, when computing allowable thumb joint torques, the intersection in Equation 3 requires us to find the intersection of a convex hull in 5D (torques for the thumb) with a 3D volume of joint torques that can be produced by contact forces at the tip of the thumb. However, the geometric approach has in fact been used in previous research to study human grasps. For example, Valero-Cuevas et al. [33] present a linear model that maps muscle activation levels to contact forces for a human hand. They inflate the Jacobian matrix to a square matrix and the specific poses they studied guarantee that the Jacobian has full rank. In this case the intersection with $\operatorname{Range}\left(J^{T}\right)$ can be avoided. Also, they only compute the maximum force capabilities of a single finger and do not include the contact friction constraints in their system.

For a general solution, it is much faster to take advantage of having a linear task description available, and compute the grasp quality metric in Equation 1 by solving a set of linear programming problems to compute each of the $w_{i, \max }$ in Equation 1.

\footnotetext{
${ }^{1}$ Note that in circumstances where $J^{T}$ has no nullspace, the space of contact forces that can be exerted on the object remains convex [35].
}

\section{Optimization Problem}

Although computing the entire space of applied wrenches can be time consuming for high DOF systems, maximizing the magnitude of an applied wrench in a given direction is efficiently solved as a linear optimization problem. Here we optimize the magnitude of the applied wrench in the given direction:

$$
\max (\alpha),
$$

where

$$
w_{i}=\alpha s_{i}
$$

with $s_{i}=\frac{t_{i}}{\left\|t_{i}\right\|}$.

Then we specify the following equality constraints:

$$
\left(\begin{array}{ccc}
-s_{i} & G & 0 \\
0 & J^{T} & -M
\end{array}\right) \quad\left(\begin{array}{c}
\alpha \\
f \\
a
\end{array}\right)=0
$$

The first row ensures that the applied wrench is in the desired direction. The second row ensures zero acceleration at the finger joints by equating torques produced by tendon activation to those required to generate the contact forces. Note that this equation is very similar to that in [18], extending that work to include tendon activation levels. Nullspaces of the problem can be analyzed in a fashion similar to that outlined in [18].

To take the friction between the finger and the object at the contact points into account to ensure that the object does not slip, we first approximate the Coulomb friction cone with a friction pyramid to keep the system linear. Let the normals of friction pyramid halfplanes of contact $j$ be $n_{j k}$, where $k=1,2, \ldots, m$ and $m$ is the number of the halfplanes in the friction pyramid, we have:

$$
n_{j k} \cdot f_{j}<0 .
$$

To compute the grasp quality metric in Equation 1, we perform the optimization problem defined in Equation 7, 9, and 10 for $i=1, \ldots, t$, setting $\left\|w_{i, \max }\right\|=\alpha$ at the end of each optimization step, and take the minimum $\frac{\left\|w_{i, \max }\right\|}{\left\|t_{i}\right\|}$ as our quality metric.

\section{RESULTS}

\section{A. Force Capabilities}

To show that hand anatomy is a factor that should be taken into account for force production, we studied four poses with the hand holding a bottle, both on a human hand model (Appendix I) and a Shadow robot hand (Appendix II), computing the forces that can be generated by each pose for task vectors along the coordinate axes. We compare these results to a model that assumes contact points are identical and independent, and we begin by describing this model. 
1) Contact points only: We can easily compute the maximum force that can be applied on the object in each direction when we do not consider the hand anatomy and the difference in finger abilities at all. Results for different hand poses are the same, because the contact points are at the same positions. For the two contact points (point ' $a$ ' and point 'b') as shown in Figure 1 (d), with a friction coefficient of $\mu$ and a maximum normal force limit of $N$, the maximum forces that can be applied are $N, N, 2 \mu N, 2 \mu N, 2 \mu N$, and $2 \mu N$ in the $\mathrm{x},-\mathrm{x}, \mathrm{y},-\mathrm{y}$, $\mathrm{z}$, and $-\mathrm{z}$ directions, respectively. The extreme forces in the directions perpendicular to the normals are equal and depend on the maximum normal force and the coefficient of friction.

2) Human hand example: Figure 1 and 2 show four human hand poses of a hand grasping a bottle. This set of poses involves two contact points. In all the poses, the contact points on the bottle are the same, which are across the bottle's diameter. For Pose A, the contact points on the hand are the distal pad of the thumb and the middle pad of the index finger. The contact points of Pose B are the distal pad of the thumb and the middle pad of the middle finger. For Pose C, they are the distal pad of the thumb and the proximal pad of the middle finger. For Pose D, the contact points on the hand are the middle side of the index finger and the distal side of the middle finger.

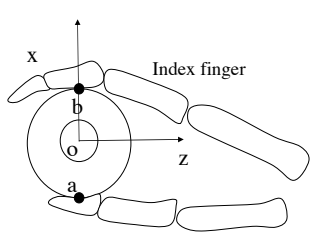

(a) Pose A

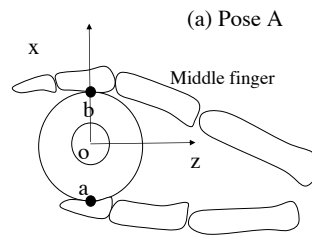

(b) Pose B

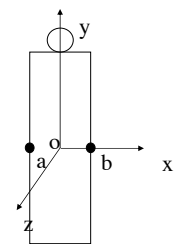

(d) Bottle Position

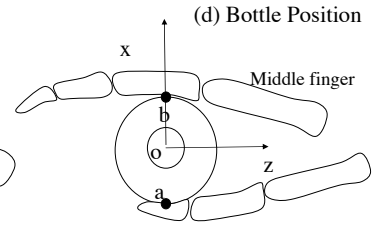

(c) Pose C
Fig. 1. Home position of the bottle and the hand poses A-C. The contact points on the hand are: Pose A. Distal pad of the thumb and middle pad of the index finger; Pose B. Distal pad of the thumb and middle pad of the middle finger; Pose C. Distal pad of the thumb and proximal pad of the middle finger.

The hand poses are captured using a Vicon optical motion capture system and then transformed to a home position of the object, where the $y$-axis points upward along the body of the object, the $\mathrm{z}$-axis points toward the hand and the $\mathrm{x}$ axis points away from the thumb. (see Figure 1 and Figure 2)

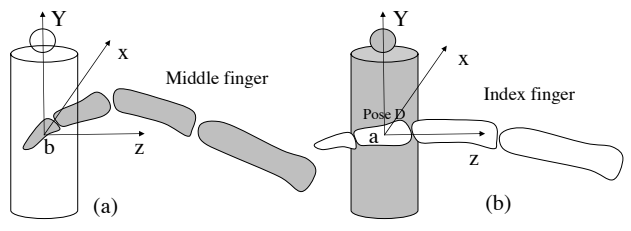

Fig. 2. Hand pose D. Contact points on the hand are: Middle side of the index finger and distal side of the middle finger. (a) and (b) are the front view of the pose with the index finger and the middle finger shown separately. Shaded areas are at the back;

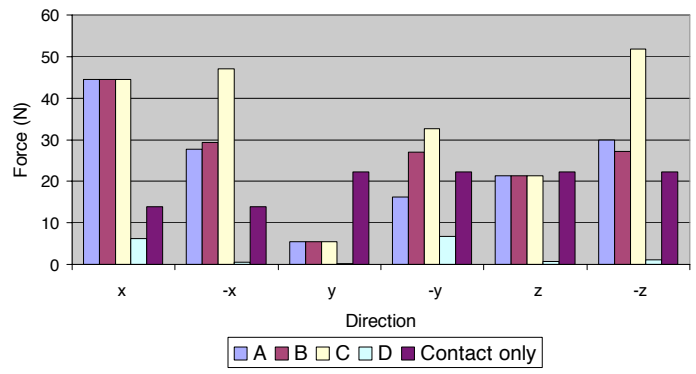

Fig. 3. Comparison of a human hand's extreme forces in the $x,-x, y,-y$, $\mathrm{z}$, and $-\mathrm{z}$ directions of the four poses grasping a bottle and the result of the abstract contact model, when the friction coefficient equals 0.8 .

To enable clearer comparison, we align the pose to the axes by removing the twist of the fingers so that in poses A-C, forces in the $\pm \mathrm{x}$ direction would be controlled mainly by the flexors and the extensors, and those on the $\pm \mathrm{y}$ axes would be controlled by the adductors and the abductors, while in pose $\mathrm{D}$, forces in the $\pm \mathrm{x}$ direction would be controlled mainly by the adductors and the abductors, and those on $\pm \mathrm{y}$ would be controlled by the flexors and the extensors. We also use the same pose for the thumbs in poses A-C, so that we could compare the poses by looking at only the differences in the other finger.

Maximum achievable forces in the $\pm x, \pm y$, and $\pm z$ direction were computed by running the optimization problem described in Equation 7, 9, and 10. Figure 3 shows a comparison of these extreme forces that the set of different poses can generate. We also plot the result of the contact only model in the same graph. To make the force ability comparable to the human hand, we use the same $\mu=0.8$ and estimate $N=13.94$ by minimizing the sum of the squared difference between the abstract contact model result and the human hand result.

Note that since the thumb, which is placed on contact point a, is in the same pose in the poses A-C, it is the pose of the finger placed on contact point $\mathbf{b}$ that makes the difference.

Overall, Pose B can produce larger forces than Pose A, because of the stronger tendon of the middle finger. Pose $\mathrm{C}$ has a better performance than Pose B, which results from the fact that the object (contact point) is brought closer to the hand (from the middle finger pad to the proximal finger 


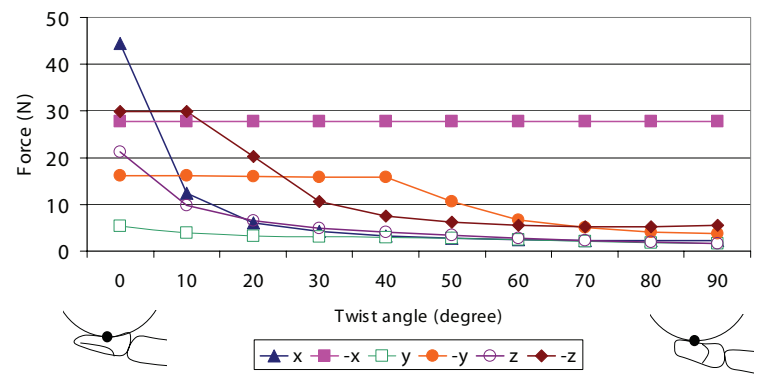

Fig. 4. Comparison of the extreme forces in $\mathrm{x},-\mathrm{x}, \mathrm{y},-\mathrm{y}, \mathrm{z}$, and $-\mathrm{z}$ directions of pose A with various twisting angles of the thumb. From 0 degrees to 90 degrees is a process of turning the thumb from facing the $\mathrm{x}$ direction as the original pose in Pose A, to facing the $-\mathrm{y}$ direction as the end position.

pad), which decreases the moment arm from the contact point to the MCP joint, for example (Figure 1 and 10). Pose D has the weakest performance among the four. For the forces on the $\mathrm{x}$-axis, fingers' adductors/abductors that produce those forces in Pose D are much weaker than the flexors/extensors, which produce the forces on the $\mathrm{x}$-axis in the poses A-C. With smaller $\mathrm{x}$-forces available and the friction cone constraints, the forces on the y-axis are very limited.

In addition to the difference in poses, asymmetries can be seen in opposing directions. The largest difference is for poses A-C in the $\pm y$ direction. The forces in the y direction are much smaller than those in the $-y$ direction, possibly caused by the equilibrium constraints and the coupling of the flexors/extensors and the adductors/abductors. For example, a tendon can flex or extend a joint and at the same time, adduct or abduct that joint. We can see from Figure 3 that the model of identical and independent contacts is not a good match to the actual capabilities of the human hand.

One important feature about the human hand is that twisting a finger's position can change the extreme force in each direction greatly, because of the strength differences in the fingers' tendons. Figure 4 shows a comparison of the extreme forces of pose A with different twisting angles of the thumb from 0 to 90 degrees. The small thumbnails illustrate the pose of the thumb at twist angle 0 and twist angle 90 .

The extreme force in the -x direction does not change, as it is only affected by the index finger. The most significant change is the drop in the force ability in the $\mathrm{x}$ direction. This is caused by the fact that the flexors (effective in the $\mathrm{x}$ direction at twist angle 0, i.e. the original Pose A.) are much stronger than the adductors (effective in the $\mathrm{x}$ direction at twist angle 90.) This leads to the drop in the maximum possible forces in the other directions as the limit in the $\mathrm{x}$ direction, together with the friction constraints, imposes a constraint in the other directions.

\section{3) Shadow hand example:}
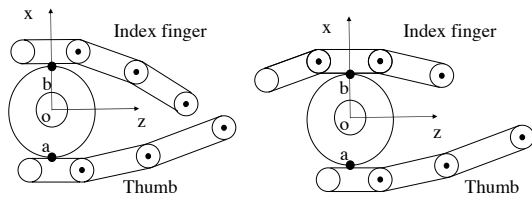

(a) Pose $\mathrm{A}$

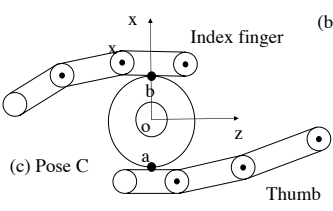

(b) Pose B

Fig. 5. Pose A-C for Shadow hand. Contact points are: Pose A. Distal pad of the thumb and distal pad of the index finger; Pose B. Distal pad of the thumb and middle pad of the index finger; Pose C. Distal pad of the thumb and proximal pad of the index finger.

Since the mechanical design of the index finger and the middle finger is the same for the Shadow hand (See Appendix II), we use the index finger for poses A-C instead of the middle finger (see Figure 5). The thumb poses are all the same for Pose A-C. Pose D remains the same as the human hand experiment. Figure 6 shows a comparison of the extreme forces that the Shadow hand can generate in the $+\mathrm{x},-\mathrm{x},+\mathrm{y},-\mathrm{y},+\mathrm{z}$, and $-\mathrm{z}$ directions and we include the contact only model with $\mu=0.8$ and maximum normal force $N=12.42$.

The result on Shadow hand has a similar pattern to the model where the contact points are considered to be identical and independent. The reason is that each of the Shadow hand's degrees of freedom is controlled by a pair of tendons, which has equal torque ability. The symmetries in design lead to the symmetric force performance on the $+y /-y$ and the $+\mathrm{z} / \mathrm{z}$ directions. The only asymmetry appears in the $+\mathrm{x} / \mathrm{x}$ direction, where the finger is more powerful than the thumb, which is caused by the stronger torque ability of the MCP flexion on the finger than the thumb. Pose D is different from the other poses, because it uses different fingers and a different pose.

As in the human hand example, we rotate the thumb of the Shadow hand from zero to 90 degrees and compare the results (Figure 7). We use Pose B of the Shadow hand experiment, which corresponds to the Pose A of the human hand experiment. The change of the force capabilities is not as dramatic as the human hand. The maximum force in the $\mathrm{x}$ direction increases and reaches a maximum at 45 degrees. One reason is that all the joints are effective and more tendons are activated: 5 tendons activated at 45 degrees and 3 activated at 90 degrees and 2 activated at 0 degrees. At 90 degrees, there is one more tendon activated than at 0 degrees and the joint torques are larger, but the moment arms from the contact point to the effective joints are also larger, which leads to a similar force ability at those two poses. 


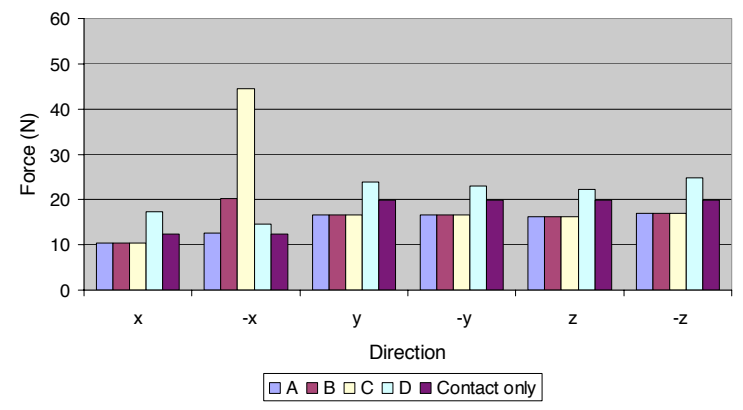

Fig. 6. Comparison of a Shadow hand's extreme forces in the $\mathrm{x},-\mathrm{x}, \mathrm{y},-\mathrm{y}$, $\mathrm{z}$, and $-\mathrm{z}$ directions of the four poses grasping a bottle and the result of the abstract contact model, when the friction coefficient equals 0.8 .

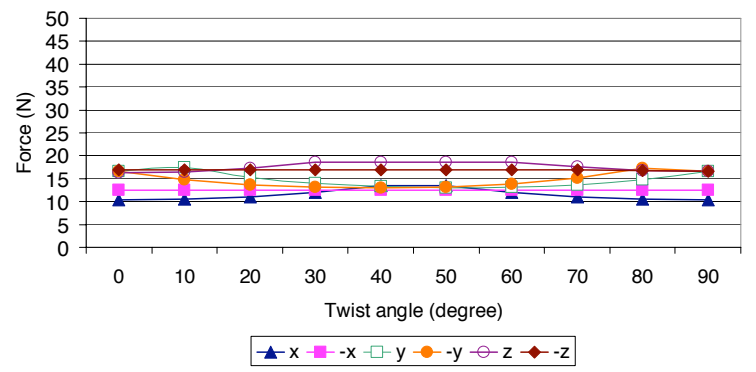

Fig. 7. Comparison of the extreme forces in the $x,-x, y,-y, z$, and $\mathrm{z}$ directions of Pose $\mathrm{B}$ with various twisting angles of the thumb. From 0 degrees to 90 degrees is a process of turning the thumb from facing the $\mathrm{x}$ direction as the original pose in Pose A, to facing the $-\mathrm{y}$ direction as the end position.

\section{B. Example of the usage of the quality metric}

Using the quality metric defined in Equation 1, we can select good grasps from different grasp candidates.

1) User defined task requirement: To compute the quality metric as defined in Equation 1, we define two tasks as the following:

a. Statically supporting the object's weight with abilities to counteract small disturbances in other directions;

b. Pushing the object downward with abilities to counteract small disturbances in other directions.

The task requirements can be described as shown in Figure 8.

2) Grasp quality metric for the four poses: Table I lists the grasp quality metric computed by Equation 1 for poses A-D and the abstract contact model for the human and shadow hand. For the human hand, pose D is of the worst quality due to the weak force ability in the $+y$ direction, while for the Shadow hand, pose D is the best because of its stronger force ability.

3) More complex grasps candidates: We have used this quality metric to select from among more complex grasps.

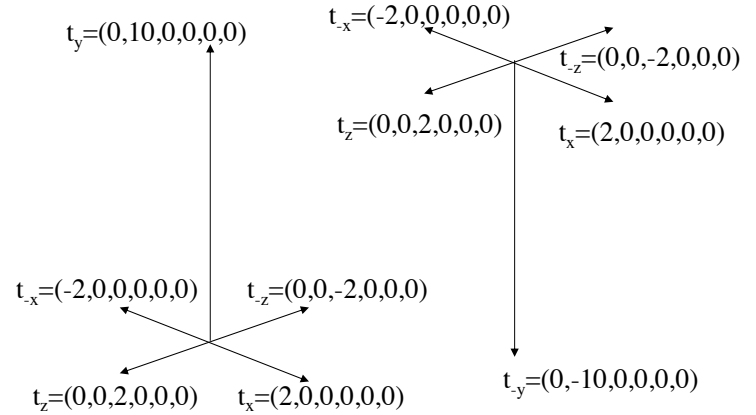

(a)

(b)

Fig. 8. Task requirements.

\begin{tabular}{|c|c|c|c|c|c|}
\hline & Pose A & Pose B & Pose C & Pose D & Abstract Contact \\
\hline Human(a) & 0.542 & 0.544 & 0.556 & 0.012 & 2.23 \\
\hline Shadow(a) & 1.656 & 1.656 & 1.656 & 2.387 & 1.987 \\
\hline Human(b) & 1.618 & 2.698 & 3.257 & 0.275 & 2.23 \\
\hline Shadow(b) & 1.656 & 1.656 & 1.656 & 2.289 & 1.987 \\
\hline
\end{tabular}

TABLE I

GRASP QUALITY METRIC BASED ON TASK REQUIREMENT (A) AND (B) FOR THE FOUR POSES AND THE ABSTRACT CONTACT MODEL FOR HUMAN AND SHADOW HAND GRASPS.

Figure 9 shows four candidates of the hand grasping a spraybottle. The quality metrics using task requirement (a) for these three grasps are 1.55, 24.3, and 13.2. The reason for the poor performance of the first candidate is that the first grasp is missing thumb contacts. Although with some contacts on the palm opposing the other fingers, the grasp is still balanced, it is weak and vulnerable to disturbances. The other two candidates both have thumb contacts and have higher quality metrics. Note that the second grasp has a larger metric, although the last two grasps have very similar contact points. The reason is that the fingers and the wrist have much stronger flexors. Grasps with the palm facing upward usually have better performance, since the forces in the y-direction are generated by the stronger flexors. Of course, other constraints such as arm comfort may make the third grasp an overall better choice in many circumstances.

\section{Discussion}

In this paper, we propose a linear programming problem to compute a grasp quality metric that takes into account the hand mechanical design and contact constraints for any task described as a polytope in wrench space. We linearize
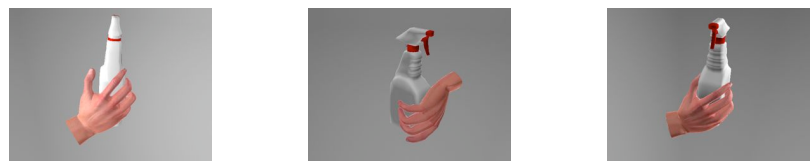

Fig. 9. Three phone grasp candidates for the spraybottle. The metrics are different because of the different contact points and hand orientation. 
the contact constraints and assume that the mechanism can actively apply the desired task wrenches.

To avoid the linear contact constraints assumption, we can set up the problem with linear matrix inequalities as in [20] with some care taken to respect activation level constraints.

Our assumption that the mechanism can always apply the desired task wrenches on the object is typically true when grasping an object subject to external disturbances. However, in other circumstances, such as analyzing net forces that can be actively applied to an object in contact with the environment, a more complex model such as that in Bicchi and Prattichizzo [28], that takes into account stiffness at the contacts would be necessary. Their approach could be adapted to maximize wrench force in a given direction and would be a more general solution than ours, but the technique presented in this paper is a much simpler solution that can be used when tendon and contact stiffness are not of primary importance.

The empirical results in this paper show that force abilities vary with finger choice and with location of the contact on the finger for both the human hand and the Shadow hand. However, while the human hand shows dramatic changes for different poses due to its asymmetric design, the Shadow hand, with a symmetric design, shows much smaller changes and resembles reasonably well the pattern shown in the results of the identical and independent contact points model. One thing we want to point out is that the test grasps we use are set up to be very symmetrical for a close match to the symmetric contact model assumption. With more complicated grasps, such as those shown in Figure 9, which use all of the fingers of the hand and the palm, have multiple contacts on each finger, and have asymmetric contact normals, we would expect to see larger changes in force capabilities for different poses for the Shadow hand.

The highly asymmetric force abilities shown in the human hand may partly explain why people use specific grasps for different tasks. One question that arises from the differences in the human hand and the Shadow robot hand results is whether there any advantage of the asymmetric force capabilities of the human hand in certain grasping or manipulation strategies.

Future work can use this framework to study this question, as well as to investigate whether people grasp objects in an optimal manner, for example, extending schemes such as that in Baud-Bovy et al. [36] to include a more accurate model of constraints on muscle forces. Another way this work can be extended is to synthesize optimal grasps when the task requirement is given and the optimal contact points on an object are computed by anatomically-based quality measures. One thing we would expect with the human hand model, for example, is a tendency to orient the fingers so that the powerful flexors can be used to apply the largest force required for a given task. Perhaps knowledge of this phenomenon could also help speed up the grasp planning process.

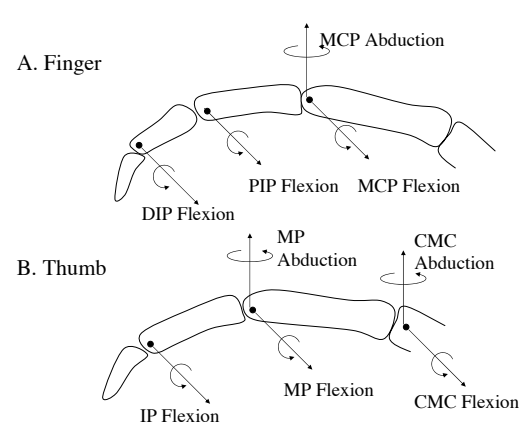

Fig. 10. A. Finger model; B. Thumb model.

\section{ACKNOWLEDGMENTS}

The authors would like to thank Jacqueline Kirchhoff, Justin Macey, Megan Monroe, Ting Ting Wu, and Ying Li for assistance in collecting the hand pose data. This research was supported by NSF awards CCF-0343161, IIS-0326322, CNS-0423546, and ECS-0325383.

\section{APPENDIX:HAND MODELS}

\section{A. Human Hand Model}

There are several hand models proposed in biomechanics ( [33], [37], and [38]). Our hand model is based on work by Valero-Cuevas et al. [33] [37] and has the following configuration (see Figure 10): The fingers have four DOF, which are three DOF of flexion/extension at the metacarpophalangeal (MCP), the proximal interphalangeal (PIP) and the distal interphalangeal (DIP) joints and one DOF of adduction/abduction at the MCP joint. The thumb has three DOF of flexion/extension at the carpometacarpal(CMC), MCP and thumb interphalangeal (IP) joint and two DOF of adduction/abduction at the $\mathrm{CMC}$ and $\mathrm{MCP}$ joints. The thumb has 8 tendons, the index finger has 7 , and the middle finger has 6 tendons. See [39] for tendon moment arms and maximum force data, which are collected from [40], [41], [42], [43]. We have verified our model by comparing maximum force capabilities to results from experiments with human subjects (see [39] for details.)

\section{B. Shadow Hand Model}

The Shadow hand model has a $2 N$ tendon-driven design. Every tendon pair controls one degree-of-freedom, except the middle and the distal joints of the fingers, which are coupled and controlled by one pair of tendons. We thus treat the fingers as having three DOF, with one DOF of adduction/abduction at the proximal joint, one DOF of flexion/extension at the proximal joint and one DOF of flexion/extension at the middle joint. The distal link and the middle link are considered as a rigid body with a small angle at the distal joint. The thumb has five DOF, which are two DOF of flexion/extension at the distal and the middle joint, two DOF of adduction/abduction at the 
middle and the proximal joint, and one DOF of rotation at the proximal joint (see [26]). See [39] for the link parameters and the maximum available joint torque data, which were obtained from [26].

\section{REFERENCES}

[1] X. Markenscoff and C. H. Papadimitriou, "Optimum grip of a polygon," International Journal of Robotics Research, vol. 8, no. 2, pp. 17-29, 1989.

[2] D. G. Kirkpatrick, B. Mishra, and C. K. Yap, "Quantitative steinitz's theorems with applications to multifingered grasping," in Proc. 20th ACM Symposium on Theory of Computing, Baltimore, Maryland, May 1990, pp. 341-51.

[3] C. Ferrari and J. Canny, "Planning optimal grasps," in IEEE Int. Conference on Robotics and Automation, Nice, France, May 1992, pp. 2290-2295.

[4] Q. Lin, J. Burdick, and E. Rimon, "A stiffness-based quality measure for compliant grasps and fixtures," IEEE Transactions on Robotics and Automation, vol. 16, no. 6, pp. 675-88, 2000

[5] B. Mishra, "Grasp metrics: optimality and complexity," in Proc. 1st International Workshop on the Algorithmic Foundations of Robotics (WAFR), Stanford, CA, 1994, pp. 137-165.

[6] V.-D. Nguyen, "Constructing force-closure grasps," International Journal of Robotics Research, vol. 7, no. 3, pp. 3-16, 1988.

[7] J. Ponce, D. Stam, and B. Faverjon, "On computing two-finger forceclosure grasps of curved 2d objects," International Journal of Robotics Research, vol. 12, no. 3, pp. 263-73, 1993.

[8] J. Ponce and B. Faverjon, "On computing three-finger force-closure grasps of polygonal objects," IEEE Transactions on Robotics and Automation, vol. 11, no. 6, pp. 868-81, 1995.

[9] J. Ponce, S. Sullivan, A. Sudsang, J.-D. Boissonnat, and J.-P. Merlet, "On computing four-finger equilibrium and force-closure grasps of polyhedral objects," International Journal of Robotics Research, vol. 16, no. 1, pp. 11-35, 1997.

[10] Z. Li and S. Sastry, "Task-oriented optimal grasping by multifingered robot hands," IEEE Journal of Robotics and Automation, vol. 4, no. 1, pp. 32-44, 1988.

[11] X. Zhu, H. Ding, and H. Li, "A quantitative measure for multi-fingered grasps," 2001 IEEE/ASME International Conference on Advanced Intelligent Mechatronics. Proceedings, pp. 213-19, 2001.

[12] X. Zhu and J. Wang, "Synthesis of force-closure grasps on 3-d objects based on the q distance," IEEE Transactions on Robotics and Automation, vol. 19, no. 4, pp. 669-79, 2003.

[13] N. S. Pollard, "Closure and quality equivalence for efficient synthesis of grasps from examples," International Journal of Robotics Research, vol. 23, no. 2, pp. 595-614, 2004.

[14] G. Liu, J. Xu, X. Wang, and Z. Li, "On quality functions for grasp synthesis, fixture planning, and coordinated manipulation," IEEE Transactions on Automation Science and Engineering, vol. 1, no. 2, pp. 14662, 2004.

[15] Y. Zhang, F. Gao, Y. Zhang, and W. Gruver, "Evaluating the quality of grasp configurations for dextrous hands," Proceedings. 1997 IEEE International Conference on Robotics and Automation, pp. 100-105, 1997.

[16] Z. Li, P. Hsu, and S. Sastry, "Grasping and coordinated manipulation by a multifingered robot hand," International Journal of Robotics Research, vol. 8, no. 4, pp. 33-50, 1989.

[17] J. Salisbury and J. Craig, "Articulated hands: force control and kinematic issues," IJRR, vol. 1, no. 1, Spring 1982.

[18] C. Melchiorri, "Multiple whole-limb manipulation: an analysis in the force domain," Robotics and Autonomous Systems, vol. 20, no. 1, pp. 15-38, 1997.

[19] J. Kerr and B. Roth, "Analysis of multifingered hands," International Journal of Robotics Research, vol. 4, no. 4, pp. 3-17, Winter 1986.

[20] L. Han, J. Trinkle, and Z. Li, "Grasp analysis as linear matrix inequality problems," IEEE Transactions on Robotics and Automation, vol. 16, no. 6, pp. 663-74, 2000.

[21] M. Buss, H. Hashimoto, and J. Moore, "Dextrous hand grasping force optimization," IEEE Transactions on Robotics and Automation, vol. 12, no. 3, pp. 406-18, 1996.
[22] J. Becker, N. Thakor, and K. Gruben, "A study of human hand tendon kinematics with applications to robot hand design," Proceedings 1986 IEEE International Conference on Robotics and Automation, pp. 1540$5,1986$.

[23] L. Barbieri and M. Bergamasco, "Nets of tendons and actuators: an anthropomorphic model for the actuation system of dexterous robot hands," 91 ICAR. Fifth International Conference on Advanced Robotics. Robots in Unstructured Environments, pp. 357-62, 1991.

[24] R. Kolacinski and R. Quinn, "Design and mechanics of an antagonistic biomimetic actuator system," Proceedings. 1998 IEEE International Conference on Robotics and Automation, pp. 1629-34, 1998.

[25] Y. Lee and I. Shimoyama, "A skeletal framework artificial hand actuated by pneumatic artificial muscles," Proceedings 1999 IEEE International Conference on Robotics and Automation, pp. 926-31, 1999.

[26] Shadow dexterous hand. [Online]. Available: http://www.shadow.org.uk/products/newhand.shtml

[27] R. Kurtz and V. Hayward, "Dexterity measure for tendon actuated parallel mechanisms," 91 ICAR. Fifth International Conference on Advanced Robotics. Robots in Unstructured Environments, pp. 1141-6, 1991.

[28] A. Bicchi and D. Prattichizzo, "Analysis and optimization of tendinous actuation for biomorphically designed robotic systems," Robotica, vol. 18, pp. 23-31, 2000.

[29] S. B. Nokleby, R. Fisher, R. P. Podhorodeski, and F. Firmani, "Force capabilities of redundantly-actuated parallel manipulators," Mechanism and Machine Theory, vol. 40, no. 5, pp. 578-99, 2005.

[30] P. Voglewede and I. Ebert-Uphoff, "Overarching framework for measuring closeness to singularities of parallel manipulators," IEEE Transactions on Robotics, vol. 21, no. 6, pp. 1037-45, 2005.

[31] E. Chinellato, A. Morales, R. Fisher, and A. del Pobil, "Visual quality measures for characterizing planar robot grasps," IEEE Transactions on Systems, Man and Cybernetics, Part C (Applications and Reviews), vol. 35, no. 1, pp. 30-41, 2005.

[32] S. Zechloul, B. Blanchard, and J. Pamanes, "Optimization of kinematics performances of manipulators under specified task conditions," in The ory and Practice of Robots and Manipulators:Proceedings of RoManSy 10, the Tenth CISM-IFTOMM Symposium, 1995, pp. 247-255.

[33] F. J. Valero-Cuevas, F. E. Zajac, and C. G. Burgar, "Large indexfinger forces are produced by subject-independent patterns of muscle excitation," Journal of Biomechanics, vol. 31, pp. 693-703, 1998.

[34] N. Pollard and R. Gilbert, "Tendon arrangement and muscle force requirements for human-like force capabilities in a robotic finger," Proceedings 2002 IEEE International Conference on Robotics and Automation, vol. vol.4, pp. 3755-62, 2002.

[35] T. Omata, "Rigid body analysis of power grasps: bounds of the indeterminate grasp force," Proceedings 2001 ICRA. IEEE International Conference on Robotics and Automation, pp. 2203 - 2209, 2001.

[36] G. Baud-Bovy, P. D., and B. N, "Does torque minimization yield a stable grasp?" Workshop on Multi-point interaction in Robotics and Virtual Reality, ICRA, pp. 1037-45, 2004.

[37] F. Valero-Cuevas, M. Johanson, and J. Towles, "Towards a realistic biomechanical model of the thumb: the choice of kinematic description may be more critical than the solution method or the variability/uncertainty of musculoskeletal parameters," Journal of Biomechanics, vol. 36, no. 7, pp. 1019-30, 2003.

[38] J. Sancho-Bru, A. Perez-Gonzalez, M. Vergara, and D. Giurintano, "A 3d biomechanical model of the hand for power grip," Transactions of the ASME. Journal of Biomechanical Engineering, vol. 125, no. 1, pp. 78-83, 2003.

[39] Data page. [Online]. Available: http://www.andrew.cmu.edu/user/jiaxinf/tendondata.htm

[40] I. Albrecht, J. Haber, and H. Seidel, "Construction and animation of anatomically based human hand models," ACM SIGGRAPH/Eurographics Symposium on Computer Animation, pp. 98-109, 2003.

[41] P. W. Brand and A. M. Hollister, Clinical Mechanics of the Hand, 3rd ed. St. Louis, MO: Mosby - Year Book, Inc., 1999.

[42] K. An, Y. Ueba, E. Chao, W. Cooney, and R. Linscheid, "Tendon excursion and moment arm of index finger muscles," Journal of Biomechanics, vol. 16, no. 6, pp. 419-25, 1983.

[43] W. Smutz, A. Kongsayreepong, R. Hughes, G. Niebur, W. Cooney, and K.-N. An, "Mechanical advantage of the thumb muscles," Journal of Biomechanics, vol. 31, no. 6, pp. 565-70, 1998. 